\title{
Active Virtual Private Network Services on Demand
}

\author{
Alex Gali ${ }^{1}$, Stefan Covaci $^{2}$ \\ ${ }^{1}$ University College London \\ United Kingdom \\ ${ }^{2}$ PopNetAgentScape \\ Germany
}

\begin{abstract}
This paper focuses on the role of the convergence of network and service management technologies in providing a support for the increased intelligence required in the provision of management solutions, and the mobility of such solutions. Such convergence benefits the interoperable services characterised by high distribution, a dynamic nature, and the complexity of used network resources. It is based on the research results achieved in the ACTS AC338 project MIAMI (Mobile Intelligent Agents for Managing the Information Infrastructure) [1], [14] and [9].

The MIAMI project focused on the use of mobility of software intelligence agents and the benefits of mobility in the context of active service management. MIAMI developed a unified Mobile Intelligent Agent framework able to meet the requirements of an emerging global open information infrastructure. Further, MIAMI created a new service management application: the Active Virtual Pipe (AVP): a dynamic Virtual Private Network Service on demand, to demonstrate the benefit of Mobile Intelligent Agent technology for network and service management.

This paper discusses the advent of mobile agent technologies and how they may enhance traditional connectivity management services in a form of Active Virtual Private Networks services, making them dynamically customisable by consumers
\end{abstract}

Keywords: Active Services, Virtual Private Networks, Virtual Enterprise, Mobile Agents, Miami Project

\section{Introduction}

A Virtual Enterprise (VE) is defined here as a temporary federation of autonomous, legally and economically independent companies that collaborate on a common business goal, taking place in electronic "virtual" space. This type of consortium is formed for a particular task, to share the knowledge, competence, resources, and business background of the participants, in sum, to contribute to the overall business goal of the VE. The federal character of the VE matches the diversity and complexity of the market offerings and products' structure. It also increases the flexibility for reacting to the rapidly shifting demands of today's markets. A VE usually consists of several partners situated at different geographical locations. The Active Virtual Pipe (AVP) provides location transparency, so that physical separation of VE members is not an obstacle to effective business activities. During the life cycle of the VE the various partners will be engaged in adding value to the other's product or service (build-up a value chain) according to the contract. A major characteristic of a VE is that all partners share the risk and the profit of the joint venture. Each member pursues the strategy of extensive "outsourcing" and concentrates on its core competencies.

Many VEs focus predominantly on information and communication based products, so there is a need for business data to be transported between the participants over an information transport network. This puts heavy demands on the underlying network infrastructure. The 
transportation of the business process data must cope with the different constraints of each individual business process. In addition, the stakeholders of a VE often belong to different network management domains as they are situated in different geographical locations. A successful VE requires a very close co-operation among all participants, as with a single company.

The above reasons highlight a VE's need for a highly dynamic and flexible information infrastructure able to provide connectivity with guaranteed quality on demand. The major drawback of conventional solutions such as the currently available virtual private networks (VPN), is their low-level adaptability to the changing requirements of the business processes, and, for the most cases they provide few QoS guarantees (e.g. bandwidth) or no guarantees at all (e.g. VPNs via the internet). The MIAMI project provides an extensive VE support environment, for the creation, operation and administration phases (including the necessary resources allocation) in an automated way. Figure 1 gives a simplified view of the proposed environment.

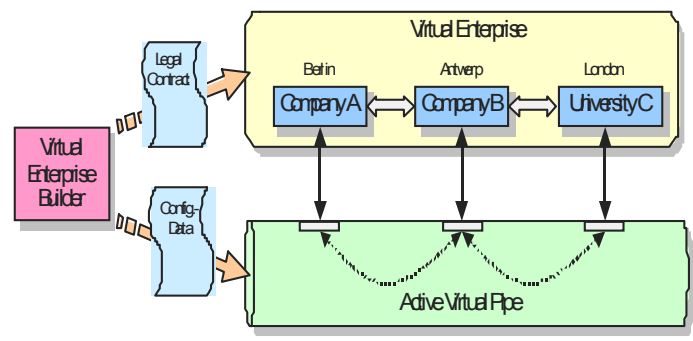

Fig. 1: A Virtual Enterprise Support Environment

\section{Active Virtual Pipe Service on Demand}

A VE usually consists of several partners situated at different geographical locations. The AVP will provide transparency between the members of the VE so as to create what is virtually a single company. The AVP is a programmable resource of the information infrastructure that supplies advanced communication and connectivity services with guaranteed QoS to the VE.

The Active Virtual Pipe (AVP) is defined as a programmable, dynamic Virtual Private Network with QoS guarantees that can be directly configured according to the demand of the VE. It as an abstract view of the dynamically (self) configurable global connectivity service that controls the transfer of telecommunication data streams. For instance, there is a possibility that the quality of service parameters of existing connections can be configured dynamically by the VE in accordance with the current business activities. Since the does not own the transport networks' resources, it makes use of the networks of several connectivity providers $(\mathrm{CP})$, which can be dynamically selected and configured on demand. Due to this capability, the AVP is in the position to offer a cost efficient, dynamic, virtual private network, well suited to the needs of VEs.

The AVP is a programmable resource of the information infrastructure that supplies a Virtual Enterprise (VE) with advanced communication and connectivity services, and a guaranteed QoS. AVP is a novel example of an Active Service [34]. It provides an abstract view of the dynamically (self) configurable global connectivity service in charge of the transfer of telecommunication data streams. It is a programmable, dynamic, QoS guaranteed, 
Virtual Private Network that can be directly configured according to the demand of the Virtual Enterprise, and it provides transparency between the members, regardless of their physical location.

Additionally, the AVP being the central "node" in the VE that represents the "glue" between all partners includes a generic workflow management facility [2], [3]. This facility can be programmed according to the needs of the business processes by the VEB. It enables an efficient, one-stop-shopping inter-organisational workflow management and provides a generic VE administration service to any VE. Furthermore the AVP allows for outsourcing of common VE management activities in order to reduce the expenditure of running a VE. Partners of the VE can focus on their own business logic and outsource the management overhead of the overall VE to the AVP. The AVP can establish a business-process-driven relationship between the service management layer and the network management layer because of its central management function and its role as an advanced connectivity provider. The AVP allows for a business process driven connectivity management.

The AVP monitors and controls the inter-organisational workflow and is able to derive appropriate the connectivity requirements used to configure the underlying network infrastructures. The workflow management facility provides the VE with a connectivity management interface at the level of workflow. The services of the AVP are provided through dynamically programmable interfaces that can be customised by the VE.

\section{Miami Design Approach}

Figure 2 shows an overview of the main components of the MIAMI system.

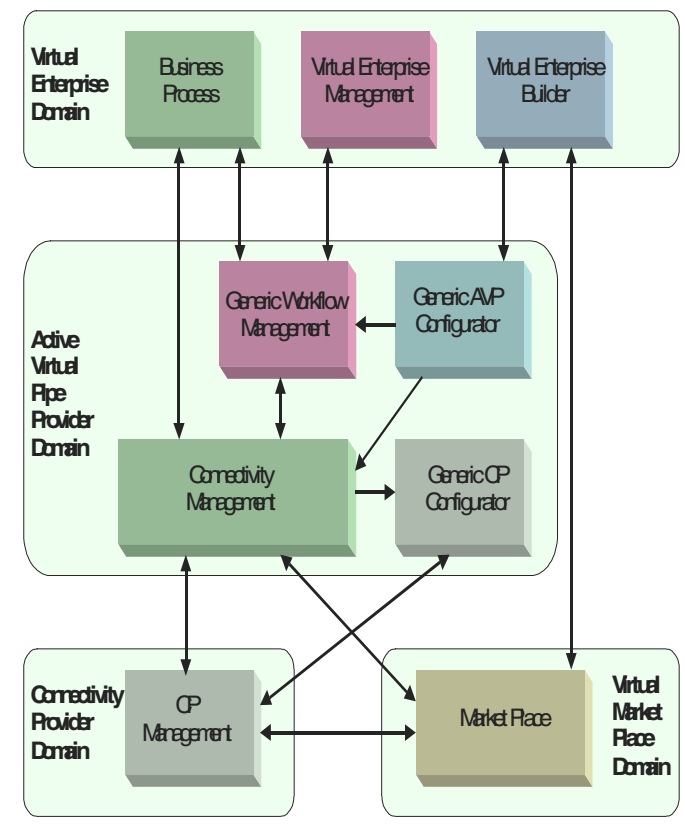

Fig. 2: MIAMI Components Overview 
VE Domain: The VE domain contains three functional facilities, the VE Builder (VEB), the VE Management and the Business Process. The VEB provides support for the definition of business processes and connectivity requirements, the search for suitable business partners including AVP Providers, negotiation among these partners, creation of the VE contract, and configuration of the selected AVP according to the contract. The finding and selection of suitable business partners and AVP Providers is achieved by the use of mobile intelligent agent technology. The configuration of the selected AVP Provider is achieved via a programmable interface. The VEB generates configuration data, which includes the definition of the business process and connectivity requirements, and programs the AVP accordingly. As a result of the configuration, a number of interfaces are instantiated and provided to the VE. These interfaces are realised by specialised dynamically created static agents that give the VE the capability to adapt the interfaces exactly to its needs.

Active Virtual Pipe Provider Domain: The AVP Provider offers the VE a programmable information infrastructure. The Generic AVP Configurator receives the configuration data from the VEB and creates and configures all runtime facilities and a Connectivity Management facility, and interfaces accordingly. The AVP Provider negotiates \& leases basic network service resources from different Connectivity Providers and provides high-level connectivity services to the VE. For the selection of the appropriate Connectivity Providers, the Virtual Market Place is searched for reasonable offers. The Connectivity Management facility and the Generic CP Configurator manage the Connectivity Providers. The VEB and Virtual Enterprise Management (VEM) together form a workflow management system.

Connectivity Provider Domain: The Connectivity Provider domain comprises one or more transport networks owned by different Connectivity Providers. Connectivity Providers give VEs the connectivity they need by supplying the Connectivity Provider Management facilities, which can be configured by the Connectivity Management facility and the Generic CP Configurator of the AVP. The Connectivity Providers (CP) manage the resources of the transport networks. The CP offers a service management interface to the AVP based on the management policies configured by the AVP Provider. The Connectivity Providers dynamically advertise on the Virtual Market Place service resources that are temporarily available, which can be bought by AVP Providers.

Virtual Market Place Domain: The Virtual Market Place domain contains a number of different market places, which can be accessed by the Subscriber, the VE Builder, the CP Management, and the Connectivity Management facility. These market places allow potential business partners, Virtual Enterprises, AVP Providers and Connectivity Providers to advertise their offers. The VE Builder searches for offers from product providers or sends its own product offers. The CPs contacts the market place to offer their services and free service resources. The AVP Provider searches the market place for reasonable CP resources, which can be dynamically allocated in order to fulfil VE's requests.

\section{Network Scenario}

The MIAMI project assumes a network scenario consisting of an end-to-end IP network which interconnects the participants of a Virtual Enterprise (VE). In addition to standard best-effort, Internet quality connectivity for general email and web browsing, the VE users also require access to higher quality connectivity facilities for real-time services such as high bandwidth video conferencing or for high speed access to large files. The users expect to pay a premium rate for guaranteed quality services, but they also wish to use lower cost, and correspondingly lower quality services for more general-purpose communications. 


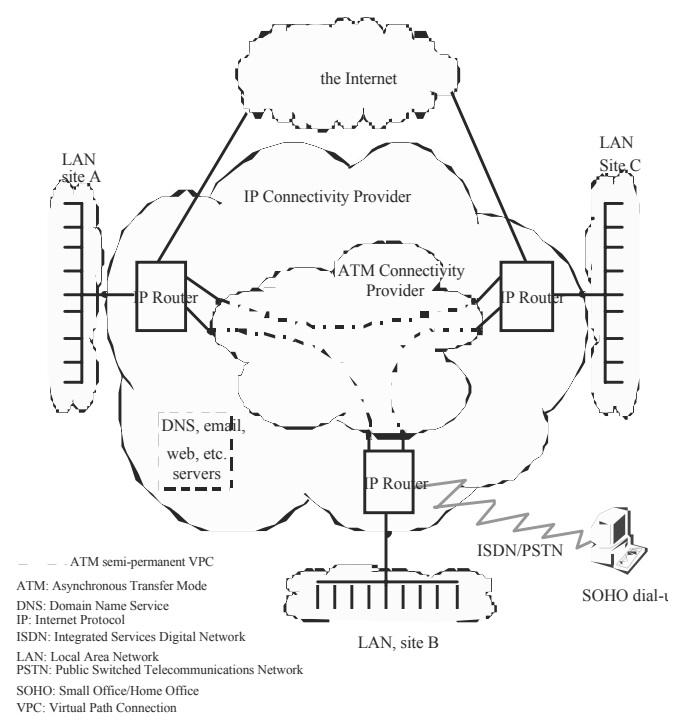

Fig. 3 Networking Scenario

In addition to providing access to the Internet, the IP Connectivity Provider (IP CP) also makes use of the services of an underlying CP - offering semi-permanent ATM connections in this case - which is able to provide guaranteed quality leased lines between the IP CP's routers. Figure 3 shows the network scenario we assume in the rest of this paper.

The service provided by the ATM CP is an end-to-end Virtual Path (VP) service offering PVPs (permanent VPs) between specified termination points. Associated with each VP are a number of parameters, which define the capacity of the connection and level of performance to be provided in terms of end-to-end delay, delay variation and tolerable cell loss ratio. VPs can be created, deleted and modified through client management actions. The clients may monitor VP usage and performance statistics, and initiate fault monitoring activities on their resources.

Issues associated with inter-administration connectivity and federation of management systems are outside the scope of this paper. The dynamic customisable approach presented for a single $\mathrm{CP}$ domain could also apply to a multiple $\mathrm{CP}$, inter-domain environment. The remainder of this paper will concentrate on the ATM $\mathrm{CP}$, although the issues discussed are also generally relevant to CPs offering managed services for any network technology.

\section{Connectivity Providers}

The responsibilities of the IP and ATM connectivity providers in terms of the network resources they manage are displayed in Figure 4. The figure shows the layered approach to AVP. Typically, responsibility for the ATM, and IP networks falls to separate organisations. Where the same organisation is responsible for both network layers, there would be a single CP.

Provider Interfaces: The ATM CP can be seen as a system with two main classes of interface:

- the interfaces to its clients (either the IP CP or the AVPP), through which it offers a set of services for the management of the connectivity; and 


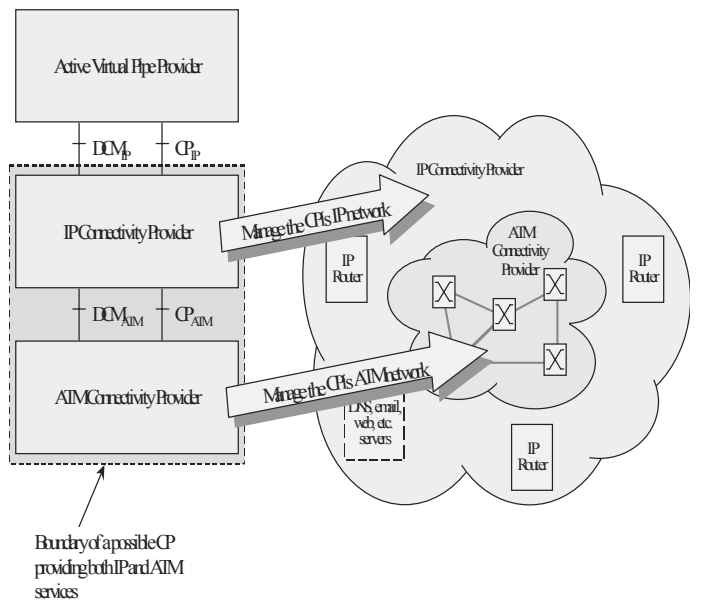

Fig. 4 Connectivity Providers Hierarchy

- the interfaces to the underlying Network Elements (NEs), which are ATM VP crossconnects in our scenario. The interfaces to the NEs will be based on the technology offered by the vendors of the switches - either SNMP or CMIP management interfaces; if CORBA-based interfaces [10], [11] are available then this is also an option. It is unlikely that switches will have mobile or intelligent agent enabled NEs in the near future.

There are two major interfaces between the ATM CP and its clients: the CP API (Connectivity Provider Application Programming Interface) and the DCM (Dynamic Connectivity Management) interface. Both of these interfaces are based on agent technology. A non-agent based approach to the design of the Connectivity Management is depicted in [17] and [15]. Agencies are made visible across each of these interfaces. These are locations where an agent may execute and may be addressed remotely. Within each agency a number of fixed, i.e. permanent, agents will be present together with a number of mobile, or visiting, agents that execute on a temporary basis. In general the fixed agents are created by the host environment- the ATM, and the clients of the ATM CP create the mobile agents.

Agent-Based Communication (ABC) refers to the mechanism by which agents communicate with one another. The term implies that specific protocols and interface definitions are used. These protocols could either be based on general distributed systems techniques for remote method invocation (CORBA or Java RMI, for example) or on higher level semantic/AI languages such as KQML [5], [12] or FIPA's Agent Communication Language (ACL) [4], [22] which support interactions with "semantic heterogeneity". The specific languages and communications protocols are dependent on the chosen agent platform and are not of direct concern in this paper. Mobile agents may communicate with fixed agents and mobile agents may communicate with other mobile agents using ABC. The communication between agents could take several forms: to raise asynchronous notifications, to query specific agents, to retrieve information and to invoke operations. A mechanism for publishing the facilities offered by an agent operating in the server role is assumed- i.e. a way of formally specifying an agent's interface. In the design of our system, the Unified Modelling Language (UML) [23] is used to specify formally an agent's interface, being subsequently mapped to the Java language. It is further assumed that an event/notification service is offered by the host environment for disseminating the events raised by agents based on filtering criteria. Today's agent platforms, including those based on OMG's MASIF specifications [8], 
do not currently offer event/notification services. In our environment it has been necessary to implement notifications in a non-generic, ad hoc basis. It should be noted that the MIAMI project is currently in the process of extending the Grasshopper agent platform [24] to allow communication between agents using FIPA's ACL.

$\mathrm{ABC}$ extends beyond the local agency to allow communication with agents in remote execution environments. This implies two methods for communications in agent systems:

either remote operations may be invoked through $\mathrm{ABC}$ (in a similar way to traditional distributed systems based on statically located objects); or

mobile agents may physically travel to the remote agency where they may run in the local environment and invoke the same operations through local (i.e. intra-node) rather than remote (i.e. inter-node) $\mathrm{ABC}$ mechanisms.

By relying on mobile agents, an active and dynamically adaptive management system can be built which is not fixed or limited by initial deployment decisions at system design or build time. The choice of using a remote or mobile agent-based communications method- is an issue, which may be decided dynamically, even at system runtime. For example, it is possible to create and deploy a mobile agent when the communications overhead between remote systems rises above a certain threshold (although this would be at the cost of physically transferring the agent to the remote execution environment).

Figure 5 shows the overall architecture of the CP's management system. The management system consists of three separate agencies for the main management activities of the CP: one for each activity: configuration management, performance management and fault management.

Configuration management: creates connection (ATM VPCs); modifies connection parameters (e.g. bandwidth); tears-down connection; maintains a database of network resources; schedules resource management and routes management.

Performance management: monitors QoS (utilisation of resources, performance of the network); logs monitored data; QoS and reports on performance.

Fault management: deals with Fault detection; Fault isolation and alarm filtering; Fault reporting; Testing

In addition there are two agencies, which represent the two classes of interface to the clients of the CP: the CP agency supports the CP interface, and the DCM agency supports the DCM interface. Within these latter two agencies a number of fixed and mobile agents may execute. The fixed agents are provided by the $\mathrm{CP}$, at initialisation time, and form the agentbased interfaces to the basic management services of the $\mathrm{CP}$. The mobile agents belong to the clients of the $\mathrm{CP}$ and are dynamically created by remote clients.

Initially, there are two main obstacles to a potential client invoking $\mathrm{CP}$ management services: legally: the client may not have a contract with the $\mathrm{CP}$ and physically: it may not have access to a management interface. The first step is to negotiate a contract. A fixed agent in the $\mathrm{CP}$ agency offers an interface to allow contract negotiation. This negotiation can be achieved in two ways: either the client creates a mobile agent to move to the CP agency and negotiate locally with the fixed agent; or the client may communicate remotely with the fixed agent.

Following successful contract negotiation, the CP creates an agency and DCM interface for the client. This involves the creation of one or more fixed agents in the DCM agency to offer specific interfaces to the management services, which feature in the contract. The fixed agents tailor (in a static sense) the management services of the $\mathrm{CP}$ to the requirements of the client and to limit access to the services according to the terms of the contract. For example, not all management services may be made available to all clients, or the geographical coverage of configuration management may be limited to specific locations. In other words, the fixed agents operate as proxys to the configuration, performance and fault management services of the $\mathrm{CP}$. 


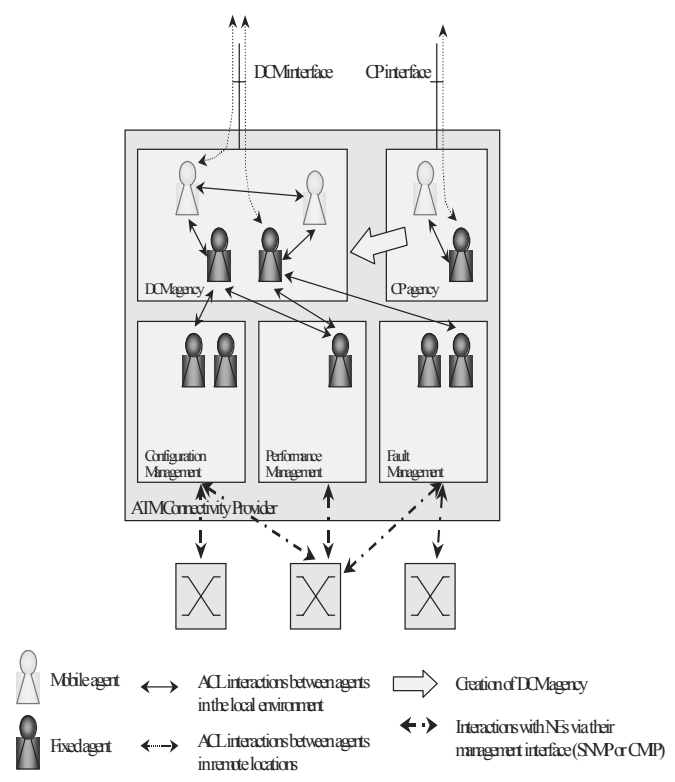

Fig. 5 Agent-based ATM Connectivity Provider

A management service could be invoked, either by a locally running mobile agent or by a remote operation from the client. When a management service is invoked, the fixed (proxy) agent triggers the corresponding operations on the agents in one of the configuration, performance or fault management agencies within the CP. It is within the latter agencies that the real management work - such as the creation of a VP - is achieved. Through the activity of the CP's configuration, performance or fault management systems, modifications are made to the network elements through their management interfaces (SNMP, CMIP) to reflect the original requests made by the clients at the DCM interface.

The MIAMI System provides a mobile intelligent agent platform which serves the needs of the enhanced European and Global Information Infrastructure defined in the ETSI Document 'Report of the Sixth Strategic Review Committee on European Information Infrastructure [35]. The MIAMI Agent platform enhances a MASIF-compliant platform by an agent communication transport service, high-level communication via ACL (agent communication language), wrappers, resource management, logging services, move transparency, and security services and, thus, builds a FIPA-compliant agent platform. These aspects are presented in more detail in [1]. This platform has been used in the development of the above scenario.

In the scenario above the interactions between the client and the CP were discussed for contract negotiation, tailoring of offered management services and dynamically invoking specific management services. This section discusses the way in which the configuration, performance and fault management systems within the CP are organised.

In general, network management systems are hierarchical with a network-wide view at the top of the hierarchy and an element-specific view at the bottom, with zero, one or more intermediate levels according to the needs of the system. This hierarchical approach can be seen in both TMN [6] and TINA [6] architectures. It is assumed that the configuration, fault and performance management systems in the agent-based CP will also follow a hierarchical architecture so as to maintain scalability and compatibility with existing management architectures and information models. 


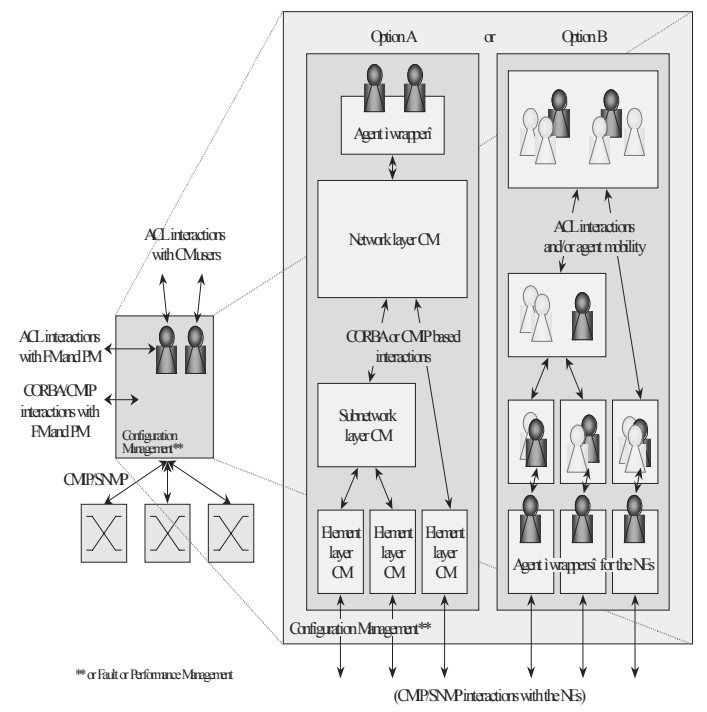

Fig. 6 Agent-based management functions

There are two ways in which the CP's management systems could be deployed:

- either through building agent wrappers on existing management software - to represent the highest level of the TMN or TINA compliant system in the agent environment; or

- through building the entire management system from scratch in an agent-based way and through building agent wrappers to represent the SNMP or CMIP interfaces of the network elements in the agent environment.

These two approaches are shown in Figure 6. Although the figure shows options for configuration management, the same holds for fault and performance management. Option A shows the approach of wrapping legacy systems with agents at the highest level; option B shows agent wrappers at the NE level and the entire system being built using agent technology.

It is also possible to take a hybrid approach. In this case, agent wrappers would be provided at each level of the hierarchy of a legacy management system. Mobile agents would then be able to visit the hierarchical level relevant to their operation and interact with fixed agents representing the legacy software at that level. Mobile agents in such a hybrid environment could relocate by traversing the hierarchy horizontally between subnetworks, or management functional areas, or they could migrate vertically to "zoom-in" or "zoom-out" to the level of detail with which they are concerned.

The hierarchical nature of management systems in TMN and TINA (option A, Fig. 6) is fixed at system design time and to a certain extent at standardisation time. For each management service to be deployed, the system designers make decisions on the placement of functionality at each hierarchical level and on whether to distribute or centralise functionality within a particular hierarchical layer. These decisions are based on many factors, including the degree of parallelism required; the quantity and complexity of information to be passed between components, the question of whether existing information models need to be modified to support the required information flows; the scalability of the solution; balancing of processing load between management workstations; the complexity of each component.

A promising application of mobile agents for network management is in deploying each management component as a set of co-operating agents (Option B in Figure 6). The way in 
which these agents are grouped and placed is initially determined by the system designers according to similar criteria as those for the design of static hierarchical TMN or TINA systems. However, now it is possible to revise the grouping and placement decisions during the operation of the management system through the mobility of agents. There are a number reasons for the migration of agents on-the-fly or for spawning new agent instances. These include: to reduce the processing load on an overloaded management workstation, to cater for an expanding set of managed resources or reduce the quantity of management traffic or information lag, between remote systems when it crosses an unacceptable threshold.

With the basic operation described in the previous section there is very little apparent advantage in adopting an agent based management system - very similar facilities are available in a traditional system based on distributed systems: TMN or TINA for example. However, there is one distinguishing advantage in the system as presented above: the way in which the DCM interface could be customised - with client-owned agents - to tailor the services offered to specific clients - this is one area where agents gain advantage over traditional software systems.

In the scenarios below mobile agents are able to autonomously interact with one or more management functional areas in the server to add value to the original management services. In traditional client-server distributed systems for network management (e.g. SNMP, TMN, TINA) the client is limited to working with the in-built facilities of the server. Any further manipulation of management information, beyond that which was generically provided by the relevant standards or by the developer of the server, must be performed in the client application code.

If customers wish to add value to the basic management services offered by the provider they must have the capability of running a management platform in their premises which supports the protocols and information models offered by the server. In addition, the customers must deploy suitable applications running on their local platform to house the required logic. The client applications must interact with the remote server to receive notifications and to initiate management operations. The quantity of information to be exchanged between client and server is a function of the management activities being undertaken and on the efficiency of the protocols and information models supported by the server for the task in hand. The delay and cost associated with each remote operation is a function of the network interconnecting the client and server. Given the particular protocols and information models supported by the server and the characteristics of the network interconnecting them, it may be not be cost effective or even possible to perform certain management tasks remotely in the client. For example, the cost of communication may outweigh the benefits of performing some management tasks (such as fine grain, real-time monitoring of performance parameters) or the information may be out of date by the time an appropriate course of action has been determined by the client application, if the network delays are too large.

With mobile agents it is possible for a client to program a mobile agent with specific functionality which may then be deployed at run-time in the server to add value above and beyond the server's basic facilities. Following deployment, the mobile agent may act autonomously to interact with the local environment and make local decisions which may then be implemented as local management actions without needing to interact with the remote client every time a decision is required. Through this approach it is possible for a remote client to deploy management behaviour and algorithms inside the remote server.

To illustrate the use of dynamically deployed agents the following three integrated scenarios we have identified in the MIAMI project:

Intelligent reporting: Mobile agents may respond to reports from both the performance and fault management systems. According to their programmed policies, rather than relaying all fault and performance reports back to the remote client, they will only report when certain 
conditions have been fulfilled. An example might be performance degradation on one connection following the failure of a connection in a remote part of the network, which forms an alternate route. Only the correlation of these two events might be relevant to the client. Alternatively, observed performance degradations might cause the agent to initiate tests to verify that unreported failures have not taken place. This scenario integrates the facilities of fault and performance management.

Fault repair: A mobile agent is programmed to listen to fault reports from the fault management system when connections have been interrupted by network failures. According to a pre-programmed policy it can initiate new connection requests between the same end points as the failed connection to restore connectivity. This scenario integrates fault and configuration management.

Bandwidth management: Assuming that performance monitoring agents have been deployed (either by the client or the bandwidth management agent itself) to monitor and report on the utilisation of connections, a bandwidth management agent will be programmed to listen to utilisation reports for certain connections. Depending on the policy for a specific connection the bandwidth management agent may decide to request increased bandwidth on highly utilised connections or to reduce the capacity of a lightly utilised connection. The decision may depend on the cost of changing the bandwidth and so a negotiation between the configuration management agents and the bandwidth management agent may take place. This scenario integrates performance and configuration management.

The integrated scenarios introduced above combine the basic facilities of the configuration, fault and performance management services offered by the server with additional customer specific logic. In other words the customer is able to program the offered management service to a certain degree. This concept has its parallel in traditional management systems through the use of the OSI management Systems Management Functions (SMFs) [25] for event forwarding and logging, resource monitoring [26] [27], and testing [28], albeit in a more limited way. Previous research work [29] [30] has demonstrated how clients can take advantage of these generic facilities to simplify the construction of intelligent clients.

We now consider how these basic facilities could be implemented through the use of mobile agents. Rather than being restricted to standardised capabilities such as the SMFs it is now possible to build entirely arbitrary and powerful behaviour into mobile agents which will be physically located in the managed system's environment. This embedded intelligence not only allows event reports tailored to the client's requirements to be emitted, but it enables the migration of the client's logic and decision-making algorithms to the server. This has an obvious impact of reducing the quantity of management traffic between remote systems and achieves more timely access to information generated by the remote server.

In OSI management, SMFs were standardised by international organisations and encapsulated in the compiled functions of OSI agents. In CORBA-based management systems the SMF-like facilities could be determined by the designers of the systems and embedded at design and system-build time. With mobile agents and intelligent reporting in agent-based management systems, the SMF-like facilities can be enhanced and extended almost infinitely and deployed at run time.

As seen in the examples above, it would be very difficult to capture such behaviour in traditional TMN or TINA systems without standardising such a bandwidth management service at the Xuser or ConS interface. If such a service was to be standardised it would be difficult to capture all possible potential behaviours that clients may request without making a comprehensive and therefore complex specification of the service in GDMO or IDL. However, this proposal allows the client to direct a dynamic and customisable network management through the use of programmable, intelligent agents based on mobile code. 


\section{Analysis and Conclusions}

Traditional and emerging frameworks for network management such as TMN and TINA allow customers electronic access to management services. However, these services are fixed in the sense that new features can only be added after a lengthy research-standardisationdeployment cycle. In this paper we have discussed the advent of mobile agent technologies and how they may enhance traditional connectivity management services making them dynamically customisable by consumers. Consumers may introduce their own value-added logic during service operation to cater for the dynamics of their environment and to enforce their own policies.

This prompts a new paradigm for building network management services. Instead of providers building services that attempt to encapsulate the requirements of all clients, they build the necessary hooks and let the clients apply their logic. Customisation and programmability of management services was always possible in traditional systems based on client-server paradigms, through the development of client applications (in the customers' premises management platform), but at high cost and low efficiency compared to the proposed agent-based approach.

The initial approach for the configuration management domain in MIAMI was to base it on an existing TMN system for ATM PVP set-up [13], [31] and [15], to which an agent interface would be added (option A in Figure 6). For logistical reasons, the final implementation is based on static agents internally (Figure 6, option B), which communicate using remote method calls. This implementation could also be based on distributed object technology e.g. CORBA. Agents were chosen for two reasons: first for uniformity, since there is no need for an adaptation agent-based interface; and second, for evaluating mobile agent platforms in the same role as distributed object frameworks. It should be finally noted that we do not see any immediate benefits from applying mobile agent technology to configuration management.

On the other hand, the performance and fault management systems use agents internally (Figure 6, option B) in a way that mobility is exercised and exploited. In the performance management domain, customised agents replace, augment and allow customisation of the functionality of the TMN/OSI-SM metric monitoring and summarisation objects [26], [27] and [16] while in the fault management domain customised agents do the same for TMN/OSI-SM testing objects [28]. In both domains, mobile agents are instantiated at the "network management level" of a management hierarchy according to requests originating from the DCM domain, migrate to network elements and perform relevant tasks locally, enjoying minimal latency and reducing network traffic. Details of the performance and fault management approaches are described in [32] and [33] respectively.

In summary, agent mobility in the presented network management architecture is used in a fashion, which we would term "weak mobility". Mobile agents are instantiated at a control point by a master static agent and then move to another point (i.e. network node) where they stay until their task is accomplished. This can be considered as an intelligent software deployment activity. The key benefit of this approach is programmability, allowing clients to "push" functionality to a point offering elementary hooks, which can be accessed to provide derived, higher-level services. In a similar fashion, we could term "strong mobility" as a situation in which a mobile agent moves from point to point using its built-in logic, adapting to changing situations in the problem domain where it is involved.

We propose a dynamic framework for creating and realising business in the information infrastructure environment. The framework capitalises on the benefits of the Mobile Intelligent Agents technology to offer an easy-to-use, automated and efficient solution to the operation of a VE across all its lifecycle phases. The framework supports customisation and as such enabling innovation in the area of future infoware products. A key mediator role of the VE framework, the Active Virtual Pipe (AVP) between the business and the service and 
network management levels has been developed. This mediator role is a novel way to integrate legacy network and service management solutions into a business driven virtual enterprise environment using Mobile and Intelligent Agent technology. AVP is a novel and key example of an Active Service. In this paper we have discussed the advent of mobile agent technologies and how they may enhance traditional connectivity management services in a form of AVP services, making them dynamically customisable by consumers.

\section{Acknowledgements}

This paper describes work undertaken in the context of the ACTS MIAMI (AC338) project. The MIAMI consortium consists of GMD FOKUS - Germany, Alcatel Bell - Belgium, Alcatel CIT - France, Algosystems SA - Greece, CRIM- Canada, France Telecom - France, Hitachi Ltd. - Japan, Imperial College of Science Technology and Medicine - U.K., University College London - U.K., The University of Surrey - U.K. The ACTS programme is partially funded by the Commission of the European Union. We would like to thank Dr Volker Tschammer, MIAMI project manager in the last part of the project, for his support.

\section{References}

1. MIAMI Project Public Web Site, http://www.fokus.gmd.de/research/cc/ima/miami/

2. Workflow Management Coalition, The Workflow Reference Model (1994)

3. Workflow Management Coalition Web Site, http://www.wfmc.org/

4. http://drogo.cselt.stet.it/fipa/

5. Labrou, Y., and Finin, T., A semantics approach for KQML - a general-purpose communication language for software agents, Third International Conference on Information and Knowledge Management (CIKM'94), November 1994.

6. ITU-T M.3010, Principles for a Telecommunications Management Network.

7. TINA consortium, Overall Concepts and Principles of TINA, Document label TB_MDC.018_1.0_94, TINA-C, February 1995.

8. Mobile Agent System Interoperability Facilities Specification OMG TC Document orbos/97-10-05, November 10, 1997. ftp://ftp.omg.org/pub/docs/orbos/97-10-05.pdf

9. MIAMI project deliverables -, http://www.fokus.gmd.de/research/cc/ima/miami/

10. CORBAservices: Common Object Service Specification", Object Management Group, 1996

11. "Common Object Request Broker: Architecture and Specification", r2.0, OMG, 1995

12. Finin, T. et. al. (1994) KQLM as an Agent Communication Language, Proc CIKM'94, ACM Press

13.Galis, A., Brianza C, Leone C, Salvatori C, Gantenbein D, Covaci, Mykoniatis G, Karayannis F Towards Integrated Network Management for ATM and SDH Networks supporting a Global Broadband Connectivity Management Service in "Intelligence in Services and Networks: Technology for Co-operative Competition", Springer- Verlag, Berlin, 1997, ISBN 3-540-63135-6

14. Galis, A., Griffin D., Eaves W., Pavlou G., Covaci S., Broos R -"Mobile Intelligent Agents In Active Virtual Pipes Support For Virtual Enterprises" In Intelligence In Services And Networks Springer Verlag, June 2000, ISBN 1586030078

15. Galis, A., -“Multi-Domain Communication Management Systems"- CRC Press, July 2000, ISBN 08493-0587-X

16. Griffin, D., Pavlou, G., Tin, T., "Implementing TMN-like Management Services in a TINA Compliant Architecture - A Case Study on Resource Configuration Management," Intelligence in Services and Networks: Technology for Co-operative Competition, ed. A. Mullery, M. Besson, M. Campolargo, R. Gobbi, R. Reed, pp. 263-274, Springer Verlag, 1997.

17. ACTS MISA AC080- Consortium and Deliverables- Management of Integrated SDH and ATM Networks, http://misa.zurich.ibm.com/ 
18. "Network Resource Architecture," The TINA Consortium, Version 3.0, Feb. 1997.

19. "Network Resource Information Model," The TINA Consortium, Document No. TB_LRS.011_2.0_94, Dec. 1994.

20. Ranc, D., Pavlou, G., Griffin, D., Galis, A., "A Staged Approach for TMN to TINA Migration", proceedings of the TINA'97 Conference, Santiago, Chile, November 1997, Proceedings to be published by IEEE

21. Kurt Rothermel, Radu Popescu Zeletin (Eds.) - Mobile Agents, First International Workshop, MA'97, Berlin, April 1997, IBSN 3-540-62803-7 Springer Verlag

22. Agent Communication Language, FIPA 97 Specification, Version 2.0, October 1998, http://www.fipa.org/spec/FIPA97.html

23. Booch, G., Rumbaugh, J., Jacobson, I., The Unified Modelling Language User Guide, AddisonWesley, 1999.

24. http://www.ikv.de/products/grasshopper/index.html

25. ITU-T Recommendations X.730-750, Information Technology - Open Systems Interconnection Systems Management Functions.

26. ITU-T X.738, Information Technology - Open Systems Interconnection - Systems Management: Metric Objects and Attributes, 1994.

27. ITU-T X.739, Information Technology - Open Systems Interconnection - Systems Management: Summarisation Function, 1994.

28. ITU-T X.745, Information Technology - Open Systems Interconnection - Systems Management: Test management function, 1994.

29. Georgatsos, P., Griffin, D., Management Services for Performance Verification in Broadband MultiService Networks, in - Bringing Telecommunication Services to the People - IS\&N'95, Clarke, Campolargo and Karatzas, eds., pp. 275-289, Springer, 1995.

30. Pavlou, G., Mykoniatis, G., Sanchez, J., Distributed Intelligent Monitoring and Reporting Facilities, IEE Distributed Systems Engineering Journal, Vol. 3, No. 2, pp. 124-135, IOP Publishing, 1996.

31. Karayannis, F., Berdekas, K., Diaz, R., Serrat, J., A Telecommunication Operators Inter-domain Interface Enabling Multi-domain, Multi-technology Network Management, Interoperable Communication Networks Journal, Vol. 2, No. 1, pp. 1-10, Baltzer Science Publishers, March 1999.

32. Bohoris, C., Pavlou, G., Cruickshank, H., Using Mobile Agents for Network Performance Management, to appear in the Proc. of the IFIP/IEEE Network Operations and Management Symposium (NOMS'00), Hawaii, USA, IEEE, April 2000.

33. Sugauchi, K., Miyazaki, S., Covaci, S., Zhang, T., Efficiency Evaluation of a Mobile Agent Based Network Management System, in Intelligence in Services and Networks - Paving the Way for an Open Service Market, Zuidweg, Campolargo, Delgado, Mullery, eds., pp. 527-535, Springer, 1999.

34. Covaci, S., (Editor) - "Active Networks"- Proceedings First International Working Conference, IWAN'99 - Berlin June 1999, Springer ISBN 3-540-66238-3

35.Report of the Sixth Strategic Review Committee on European Information Infrastructure, www.etsi.org/specrec/SRC6/SRC6.htm 